\title{
From interpretation to segmentation
}

\author{
ARNO KONING and ROB VAN LIER \\ Radboud Universiteit Nijmegen, Nijmegen, The Netherlands
}

\begin{abstract}
In visual perception, part segmentation of an object is considered to be guided by image-based properties, such as occurrences of deep concavities in the outer contour. However, object-based properties can also provide information regarding segmentation. In this study, outer contours and interpretations of object configurations were manipulated to examine differences between image-based and object-based segmentation in a visual search task. We found that locating a two-dimensional object configuration with deep concavities in the outer contour depends on the type of outer contour of the surrounding distractors. In addition, locating a three-dimensional object configuration was harder when it was surrounded by object-based-disconnected distractors, as compared with object-based-connected distractors, regardless of image-based connections in these distractors. We conclude that segmentation based on the outer contours of a target facilitates its localization. However, when three-dimensional information is available, segmentation strongly depends on object-based properties, rather than on image-based properties.
\end{abstract}

In visual perception, segmentation is the process of dividing proximal stimulations into separate objects. Several approaches to segmentation that the visual system may take have been proposed. Among these are segmentation based on concavities in the outer contour (Hoffman \& Richards, 1984; Hoffman \& Singh, 1997), segmentation based on necks versus limbs (Siddiqi, Tresness, \& Kimia, 1996), and segmentation based on the so-called shortcut rule (Singh, Seyranian, \& Hoffman, 1999). However, the relative ease with which the visual system seems to generate interpretations of objects leads to the question of whether not only image-based (IB) properties, such as those employed in the approaches above, but also objectbased $(\mathrm{OB})$ properties play a role in segmentation. In this study, we investigate the differential role of IB versus OB properties in the segmentation of objects.

Consider the objects presented in Figure 1. In Figure 1A, the locations of segmentation based on, for example, concavities in the outer contour are indicated by the arrows. In this case, the segmentation process results in the perception of one object with two protrusions. Note that, any IB approach to segmentation would result in the same segmentation of the object as that presented in Figure 1A. In Figure 1B, the same outer contour is presented, but now inner junctions (i.e., the intersections of

This research was conducted at the Nijmegen Institute for Cognition and Information. R.L. received a grant from the Royal Netherlands Academy of Arts and Sciences. The authors thank Ron Rensink and an anonymous reviewer for their helpful comments and suggestions on a previous draft of this article. In addition, the authors thank Tessa de Wit for her helpful comments and suggestions and Hubert Voogd for programming the experiment. Correspondence concerning this article should be addressed to A. Koning, Lab. Experimentele Psychologie, Tiensestraat 102, B-3000 Leuven, Belgium (e-mail: arno.koning@ psy.kuleuven.be). line segments that are not part of the outer contour) are also drawn. Again, the locations of segmentation based on the outer contours are indicated, as well as the locations of segmentation based on the inner junctions. Figure $1 \mathrm{~B}$ is readily perceived as showing an object with one protrusion and with a second object in front of it. Note that segmentation based on outer contours does not have to be incorrect when inner junctions are made visible. After all, on the basis of the outer contour in Figure 1A, the likelihood of perceiving a single object with two protrusions is higher than the likelihood of perceiving two objects. That is, for two objects (as represented in Figure 1B) to result in the outer contour presented in Figure $1 \mathrm{~A}$, requires a highly accidental positioning of the objects. The example is given only to illustrate the fact that the interpretation of stimuli (on the basis of OB properties) can result in one's perceiving separate objects although the outer contour remains the same.

To investigate differential effects in the processing of visual features such as IB and OB properties, a visual search task can be used. In a visual search task, a target has to be detected among an increasing number of distractors (Treisman \& Gelade, 1980). The target can, for example, differ from the distractors on the basis of a single stimulus property. When this target is detected equally quickly among an increasing number of distractors, the slope of search time as a function of display size will be low or, perhaps, close to zero. Alternatively, when the stimulus property in question is present only in the distractors, but not in the target, search time may, for example, increase with an increasing number of distractors. In this case, the slope of search time will be high (or at least, higher than in the opposite situation). The comparison of these two slopes is then referred to as a search asymmetry, which indicates that the presence of the stimulus property in question can be detected relatively early in the visual 


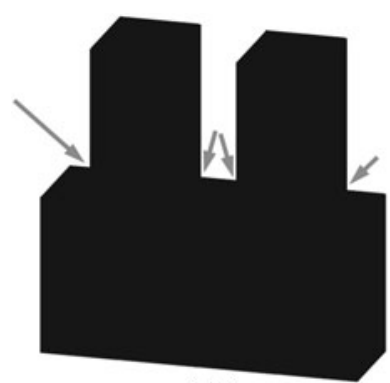

(A)

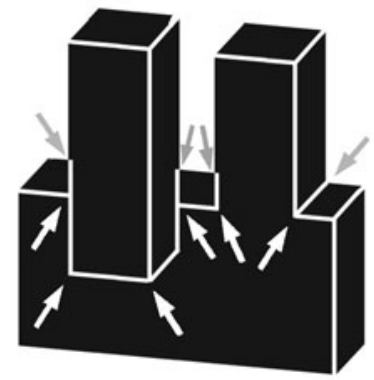

(B)

Figure 1. (A) An object for which only the outer contour is shown. The arrows indicate the locations of segmentation based on deep concavities in the outer contour. (B) The same outer contour as that in panel $A$ is presented, but now inner junctions are also drawn. The arrows indicate the locations of segmentation of the object based on both the outer contours and the inner junctions.

process. Should the slopes be identical to each other, there is no search asymmetry, and the stimulus property in question is said to be processed relatively late. Using a visual search task to investigate segmentation based on outer contours, various authors have found that this type of
(IB) segmentation is a relatively early process (Hulleman, te Winkel, \& Boselie, 2000; Kunar, Humphreys, Smith, \& Hulleman, 2003; Vecera, Behrmann, \& Filapek, 2001; $\mathrm{Xu} \&$ Singh, 2002). However, it has been suggested that OB properties are among the first stages of threedimensional (3-D) object recognition as well (e.g., Biederman, 1987). Indeed, Enns and Rensink (1990) found that a 3-D object, placed in a particular orientation, is detected relatively quickly among $180^{\circ}$-rotated 3 -D objects (i.e., only the orientation of the inner junctions of the 3-D objects differed, whereas the outer contour remained the same), in contrast to similar two-dimensional (2-D) objects. Since 3-D stimulus properties have been found to be processed relatively quickly, it has been suggested that these OB properties might be processed earlier than previously had been thought (Enns \& Rensink, 1990; Palmer, 1999), which may indicate that segmentation processes based on OB properties are also processed earlier than previously had been thought.

Regarding the segmentation of proximal stimulations into multiple 3-D objects, an important aspect is the connectedness between the objects (van Lier \& Wagemans, 1998). Connectedness between 3-D objects can be described on at least two levels: $O B$ connectedness refers to connections between objects in 3-D space; IB connectedness refers to connections between objects in the image. Recent research has shown that OB connectedness is more of a determining factor than is IB connectedness (Koning \& van Lier, 2003, 2004; Saiki \& Hummel, 1998). Consider now the 3-D configurations presented in Figure 2. The 3-D configuration that is indicated by full contour segmentation shows two IB-/OB-disconnected objects. The 3-D configuration that is indicated by intermediate contour segmentation shows two objects that are OB disconnected but IB connected. Note that there are

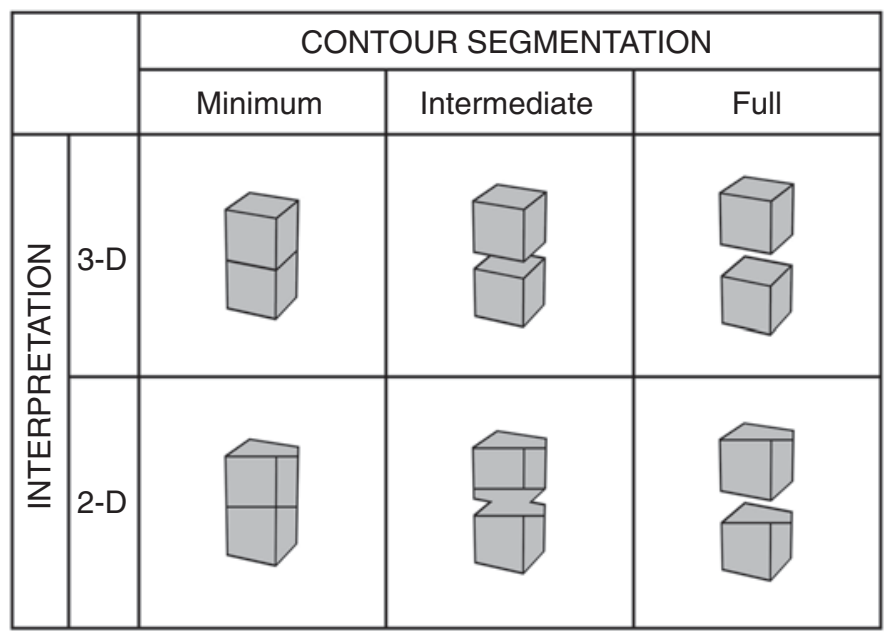

Figure 2. The configurations used in the experiment, which were designated by the degree of contour segmentation and by their interpretation. Contour segmentation comprised three levels: minimum, intermediate, and full. Interpretation comprised two levels: three-dimensional (3-D) and two-dimensional (2-D). 
no additional depth cues available (such as shadows or stereoscopic information) that indicate whether the two cubes are, in fact, disconnected in 3-D space. However, for this configuration, an interpretation of OB disconnectedness is more likely than an interpretation of $\mathrm{OB}$ connectedness. As a result, just on the basis of the outer contours, a configuration showing full contour segmentation is expected to be detected more quickly in a visual search task when it is surrounded by configurations showing intermediate contour segmentation, as compared with the opposite situation. In addition, when a configuration showing intermediate contour segmentation is surrounded by configurations showing minimum contour segmentation, search performance should also be easier, as compared with the opposite situation. In other words, search performance is expected to be influenced by the level of contour segmentation. Nevertheless, on the basis of the interpretations of these stimuli, such findings would contradict previous findings regarding $3-D$ objects and OB versus IB connectedness (Koning \& van Lier, 2003, 2004; Saiki \& Hummel, 1998), since it has been argued that OB properties are more of a determining factor than are IB properties.

In the present study, outer contours and interpretations of configurations were manipulated in order to examine differences between IB and OB segmentation in a visual search task. Three 3-D configurations were used. The spatial relation between two objects was varied for each configuration, resulting in different outer contours on the basis of which OB segmentation was possible. In addition, comparable 2-D configurations were created. On the basis of earlier research (Koning \& van Lier, 2003, 2004; Saiki \& Hummel, 1998), we expected that for the 3-D configurations, OB (dis)connectedness would be more important than IB (dis)connectedness for identifying targets in a visual search display. Alternatively, if IB segmentation is, indeed, a relatively early process (e.g., Hulleman et al., 2000; Xu \& Singh, 2002), as compared with OB segmentation, we would expect that the IB properties would help in the identifying of targets in a visual search display, regardless of the OB properties. With respect to the 2-D configurations, we expected that target identification would rely more on IB segmentation.

\section{METHOD}

\section{Participants}

Fourteen participants (18-31 years of age) performed the experiment and were given course credit or were paid for their time. All the participants had normal or corrected-to-normal vision.

\section{Stimuli}

In total, there were six configurations (see Figure 2). First, three 3-D configurations were created. Each configuration consisted of two identical cubes. In the first configuration, the cubes were both $\mathrm{OB}$ and IB connected. In the second configuration, the cubes were OB disconnected but IB connected. In the third configuration, the cubes were both OB and IB disconnected. Second, three 2-D configurations were created by modifying the inner junctions of the 3-D configurations (see Figure 2), to change the interpretations of the objects. Structural changes of the configurations were mini- mized, although still establishing a 2-D character for each configuration. The interpretation variable thus consisted of two levels: 3-D and 2-D.

The contour segmentation variable comprised three levels: minimum, intermediate, and full. With respect to minimum contour segmentation, on the basis of the outer contour alone, the configuration was not segmented into two separate objects. For the intermediatelevel contour segmentation, due to the two concave angles in the outer contour, segmentation of the configuration into two separate objects was possible. In the third level, full contour segmentation, there were two outer contours present in the configuration.

In the visual search task, each of the six configurations could be a target or a distractor. When a 3-D configuration was the target, the distractors were other 3-D configurations. Similarly, when a 2-D configuration was the target, the distractors were other 2-D configurations. Thus, in total, there were 12 target-distractor combinations: For both the 3-D and the 2-D configurations, six target-distractor combinations were possible (i.e., each level of contour segmentation could be a target, whereas the remaining two levels could be distractors).

For each target-distractor combination, target displays were created as follows. A white screen was divided into four same-sized quadrants. Each quadrant contained a $3 \times 2$ imaginary grid. First, a target was placed randomly in a cell of the imaginary grid of one quadrant. Next, a distractor was placed randomly in a cell of the imaginary grids for each of the remaining three quadrants. This resulted in a target display with a total display size of four elements. The targets could appear equally often in one of the four quadrants. This procedure was performed for all the target-distractor combinations. Display sizes with 8,12 , and 16 elements were made by following the same procedure, but now one, two, or three additional distractors were randomly placed in each imaginary grid of each quadrant. When all the elements had been placed in a target display, each element was randomly jittered (both horizontally and vertically) by a maximum of 15 pixels ( 20 arcmin). This was done to avoid grouping of the elements in rows or columns. An example of a target display with 8 elements is shown in Figure 3A. A total of 192 trials were created: 12 target-distractor combinations, four display sizes, and four quadrants in which a target could appear.

\section{Procedure}

The experiment was performed at Radboud University. The participants were seated $1 \mathrm{~m}$ from the screen, with their heads stabilized by a chinrest. The displays subtended a visual angle of $15^{\circ} \times$ $11^{\circ}$. Each element subtended a visual angle of less than $30 \mathrm{arcmin}$ vertically and 15 arcmin horizontally. After the procedure had been explained and the possible target-distractor combinations had been shown, a practice task was given.

Figure 4 shows a schematic representation of the timeline of events. Each trial started with a fixation cross $(750 \mathrm{msec})$. Next, a target-display was shown. As soon as the participant identified the target, a button had to be pressed. Half of the participants used their dominant hand to give the response; the other half used their nondominant hand. Reaction times (RTs) were measured to the nearest millisecond. Next, a white screen appeared (500 msec), after which a response display was shown. Response displays were created by replacing the target in the target display with a distractor of the same kind as those already present. An example of a response display with eight elements is shown in Figure 3B. The white screen was added in order to prevent identification of the target through apparent motion as the target was replaced by a distractor. In addition, because the target in the response display was replaced by a distractor of the same kind, the participants were prevented from employing a strategy of continuing to search for the target after the first reaction had been given. In the response display, a red rectangular outline was positioned around the element in the upper left corner of the display. The participant had to press a button to navigate this rectangle through all the elements in the response display 


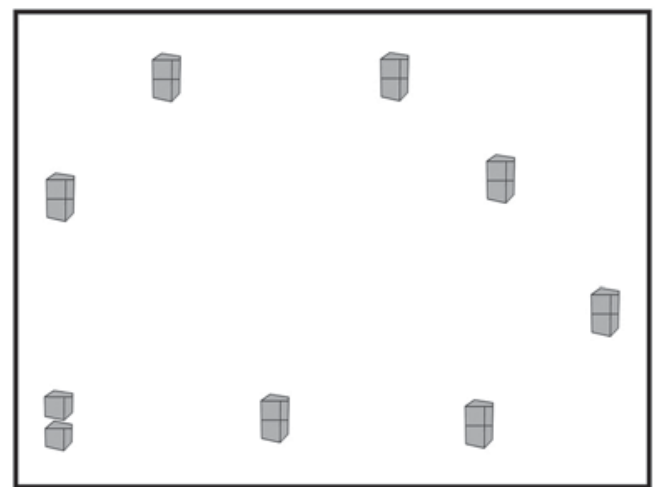

(A)

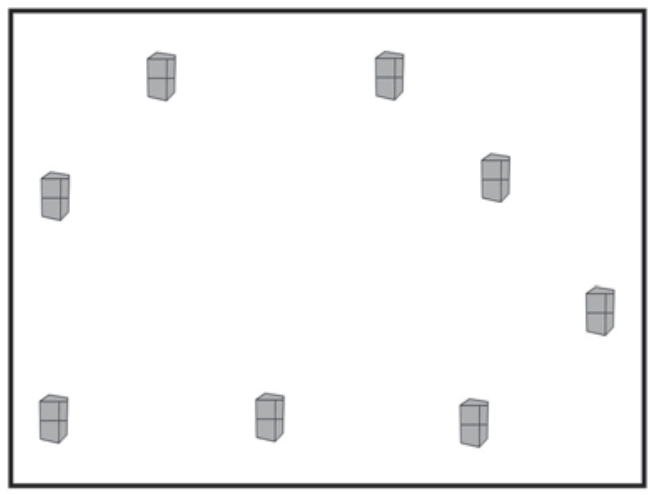

(B)

Figure 3. (A) Example of a target display with eight elements. A two-dimensional (2-D) configuration with full contour segmentation is the target; the distractors are 2-D configurations with minimum contour segmentation. (B) Example of a response display, in which the target shown in Figure 3A has been replaced by a distractor of the same kind as the one already present in Figure $3 \mathrm{~A}$.

to the location where the target had been seen. After the rectangle had been positioned around the element that was identified as the target in the target display, a button had to be pressed to validate the decision. All the trials were presented randomly to each participant in a single session. A random sample of 10 trials was used as a practice task.

\section{RESULTS}

There was a $2 \%$ error rate across all participants. The RT data were analyzed using the procedure in Lorch and Myers (1990). For each participant, the slopes (i.e., search

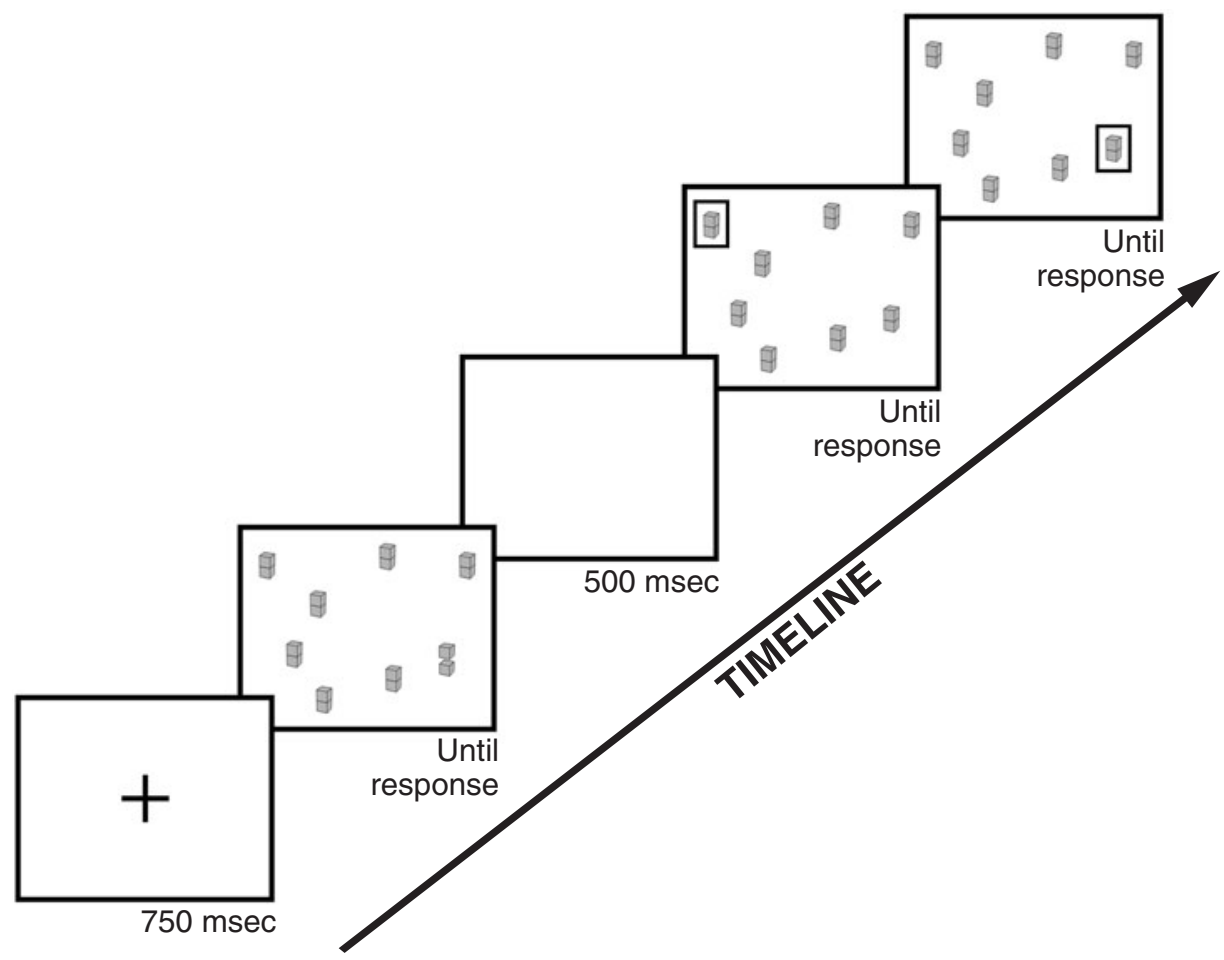

Figure 4. Schematic representation of the timeline of events in the experiment. After presentation of a fixation cross $(750 \mathrm{msec})$, a target display was shown. The target display remained on the screen until a response had been given. After the response, a blank screen appeared $(500 \mathrm{msec})$, followed by a response display. In the response display, the target was replaced by a distractor, and a red rectangular outline was positioned over the configuration in the upper left corner of the display. The participant had to navigate the outline until it was positioned over the configuration where the target had been seen. With a final response, the perceived location of the target was recorded. 
rates) of all 12 target-distractor combinations were calculated using simple regression analyses. Next, to examine search asymmetries, the search rates of corresponding target-distractor combinations were entered into a repeated measures ANOVA for both the 3-D and the 2-D configurations. This resulted in three different repeated measures ANOVAs: one for the target-distractor combination with minimum versus intermediate contour segmentation, one for the combination with minimum versus full contour segmentation, and one for the combination with intermediate versus full contour segmentation. Consequently, each ANOVA was two factorial, with interpretation (2), and target-distractor combination (2) as the independent variables and search rate (in milliseconds/item) as the dependent variable. Figure 5 shows the means of the search rates as a function of the target-distractor combination and interpretation variables.

In the first ANOVA (minimum vs. intermediate contour segmentation; Figure 5A), only the interaction between target-distractor combination and interpretation was significant $[F(1,13)=4.92, p<.05]$. Contrast comparisons revealed no differences between the 2-D configurations $(F<1)$. For the 3-D configurations, the search rates for displays that showed targets with minimum contour segmentation were larger than those for displays with targets that showed intermediate contour segmentation $[F(1,13)=7.89, p<.05]$. Thus, a search asymmetry was found for the 3 -D configurations, indicating that when a target with minimum contour segmentation had to be searched for among distractors that showed intermediate contour segmentation, search was significantly harder, as compared with the opposite situation.

In the second ANOVA (minimum vs. full contour segmentation; Figure 5B), a main effect was found for the target-distractor combination variable $[F(1,13)=50.11$, $p<.001]$. The search rates for the target-distractor combinations that showed targets with minimum contour segmentation and distractors that showed full contour segmentation were significantly larger than those for the target-distractor combinations that showed the opposite with respect to contour segmentation. That is, a search asymmetry was found for the 2-D configurations $[F(1,13)=22.22, p<.001]$, as well as for the 3 -D configurations $[F(1,13)=38.88, p<.001]$.

In the third ANOVA (intermediate vs. full contour segmentation; Figure 5C), a main effect was found for the variable interpretation $[F(1,13)=15.00, p<.005]$. The 2-D configurations had significantly smaller search rates than did the 3-D configurations. For both types of configurations, no search asymmetries were found.
(A)

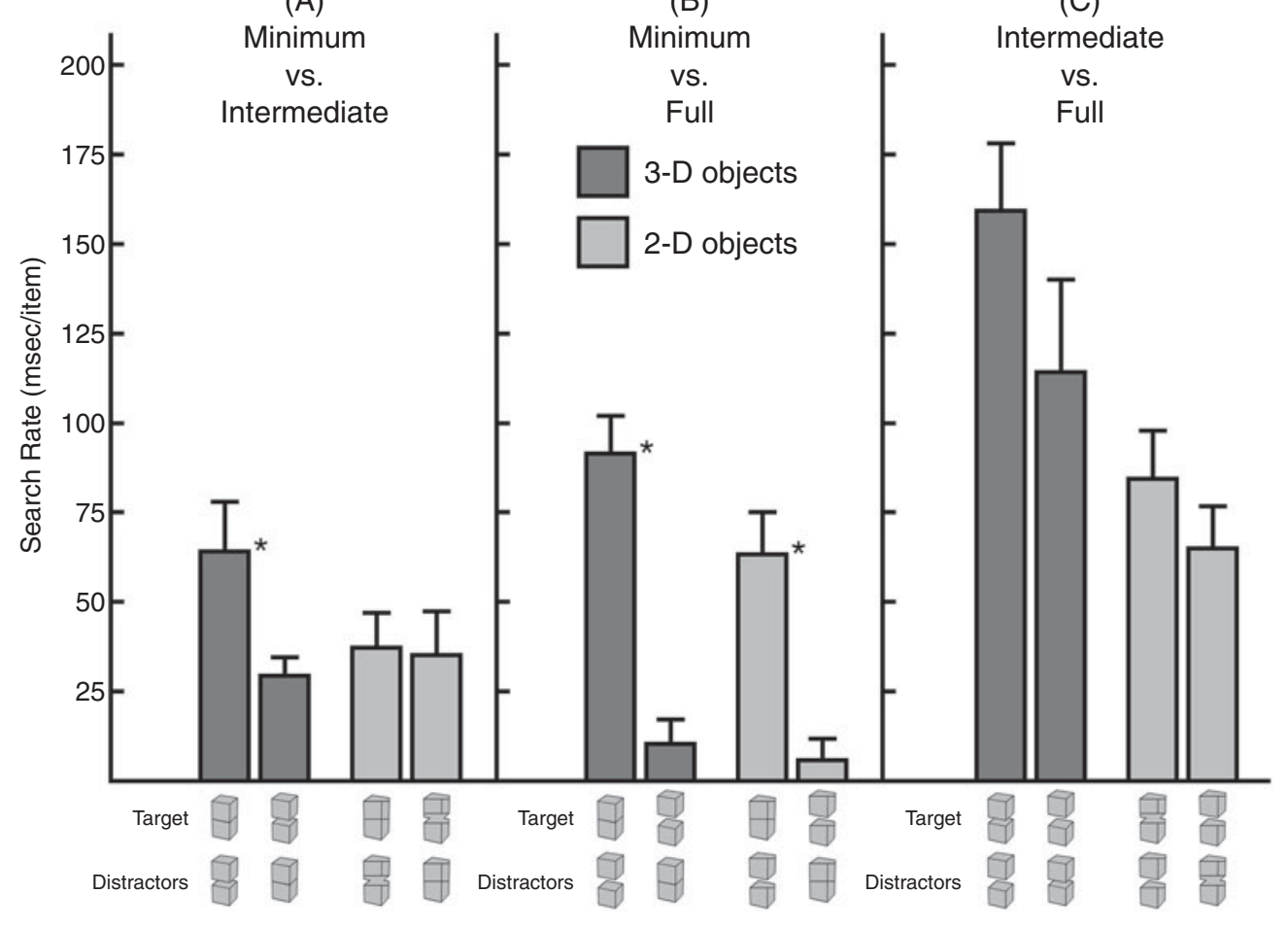

Figure 5. Bar graphs of the mean search rates (in milliseconds/item) as a function of target-distractor combination and interpretation. (A) Target-distractor combination with minimum versus intermediate contour segmentation. (B) Target-distractor combination with minimum versus full contour segmentation. (C) Target-distractor combination with intermediate versus full contour segmentation. Error bars represent one standard error of the mean. An asterisk placed between a pair of bars indicates a significant difference (i.e., a search asymmetry) between the corresponding target-distractor combinations. 


\section{DISCUSSION}

For the 3-D configurations, two search asymmetries were found. As can be seen in Figures 5A and 5B, when the distractors were 3-D configurations that showed either intermediate or full contour segmentation, search was relatively hard. One might argue, thus, that when a target is surrounded by, for example, 15 configurations showing intermediate or full contour segmentation, the number of interpreted elements on the screen is more than 15 . Such an interpretation could consequently decrease search performance. It is not suggested that this is in fact the case, since the configurations showing full contour segmentation are still grouped by, for example, the Gestalt principle of proximity. However, this does show that identifying targets depends on the $\mathrm{OB}$ (dis)connectedness of the elements in a visual search display. No search asymmetry was found between 3-D configurations showing intermediate and full contour segmentation (Figure 5C). Apparently, additional IB disconnectedness between 3-D configurations does not further decrease search performance, as compared with IB connectedness between 3-D configurations, provided that an interpretation of $\mathrm{OB}$ disconnectedness is possible. Even though the 3-D configurations showing intermediate contour segmentation do not necessarily have to be OB disconnected, they do seem to result in an interpretation of two separate objects. Once an interpretation of two OB-disconnected 3-D objects can be made, it is hard to let this interpretation go and take advantage of additional IB qualities that may be present in the display. These results are in line with previous work regarding IB/OB connectedness and 3-D objects (Koning \& van Lier, 2003, 2004; Saiki \& Hummel, 1998). In addition, the present results are in line with the suggestion of both Enns and Rensink (1990), and Palmer (1999) that 3-D stimulus properties may be processed earlier than has been previously thought. Nevertheless, the results of the 3-D configurations need to be contrasted with those of the 2-D configurations, since the results might have been due to different levels of contour segmentation, and not to their interpretation.

For the 2-D configurations, one search asymmetry was found (see Figure 5B) for the configurations showing minimum versus full contour segmentation. This seems to imply that IB disconnectedness between the image fragments is sufficient for locating a target. However, the findings of the 3-D configurations, as well as the findings by Koning and van Lier $(2003,2004)$, show that when 3-D object properties are available, these properties can overrule IB properties. Since no other search asymmetries were found, it must be noted that the 2-D configurations showing intermediate contour segmentation are not segmented when they are surrounded by 2-D configurations showing minimum contour segmentation (Figure 5A) but that they are segmented when they are surrounded by 2-D configurations showing full contour segmentation (Figure 5C). The 2-D configuration showing intermediate contour segmentation thus seems to be an ambiguous figure with respect to segmentation. Whether this configuration is segmented seems to depend on the context in which it is shown. Note that this suggestion implies that the configurations showing intermediate contour segmentation can be influenced by the other configurations. It is not suggested that, when the configurations showing intermediate contour segmentation are distractors, these configurations can influence segmentation of the other configurations. The possibility of the influence of context in the visual search paradigm has been put forward in different studies (e.g., Brown, Weisstein, \& May, 1992; Duncan \& Humphreys, 1989; Rauschenberger, Peterson, Mosca, \& Bruno, 2004). It was argued by Duncan and Humphreys that when the diversity between the target and the distractors decreases, search rate increases. Thus, when the interpretation of the target regarding segmentation into two separate objects is ambiguous, the kind of distractors that are present in the display may influence search performance.

Considering the stimuli, it must be noted that the 3-D and the 2-D configurations showing intermediate contour segmentation differ not only in their interpretation, but also in their type of connections. That is, the 3-D configurations are separated by T-junctions, whereas the 2-D configurations are separated by L-junctions (i.e., the L-junctions at the locations of the concavities in the outer contour). T-junctions can trigger an occlusion interpretation (Rensink \& Enns, 1998) and, thus, also a (3-D) depth interpretation. Therefore, replacing the L-junctions with T-junctions for the 2-D configurations that show intermediate contour segmentation can lead to results similar to those for the 3 -D configurations, because of a segmentation of surfaces in depth. We performed a control experiment in which such a configuration was used. The stimuli and the results for the control experiment are shown in Figure $6 \mathrm{~A}$. Note that the term intermediate contour segmentation has been replaced by the term $T$-junction. The configuration showing T-junctions is easily interpreted as consisting of two partly overlapping hexagonal surfaces. The same task was administered $(N=14)$, and the same data analysis was performed. Search asymmetries were found between configurations showing minimum contour segmentation and those showing T-junctions $[F(1,13)=59.33, p<.001$; Figure $6 \mathrm{~A}$, two bars on the left] and between configurations showing minimum and those showing full contour segmentation $[F(1,13)=22.52$, $p<.001$; Figure 6A, two bars in the middle]. No search asymmetry was found between configurations showing T-junctions and those showing full contour segmentation $(p>.1$; Figure 6A, two bars on the right).

As was expected, the results were identical to those for the 3-D configurations in the main experiment. In other words, an occlusion interpretation (triggered by Tjunctions) can also lead to OB segmentation. However, the question now remained as to whether the local T-junctions by themselves are sufficient. Therefore, another control experiment was performed, of which the stimuli and results are shown in Figure 6B. As can be seen in Fig- 
(A)

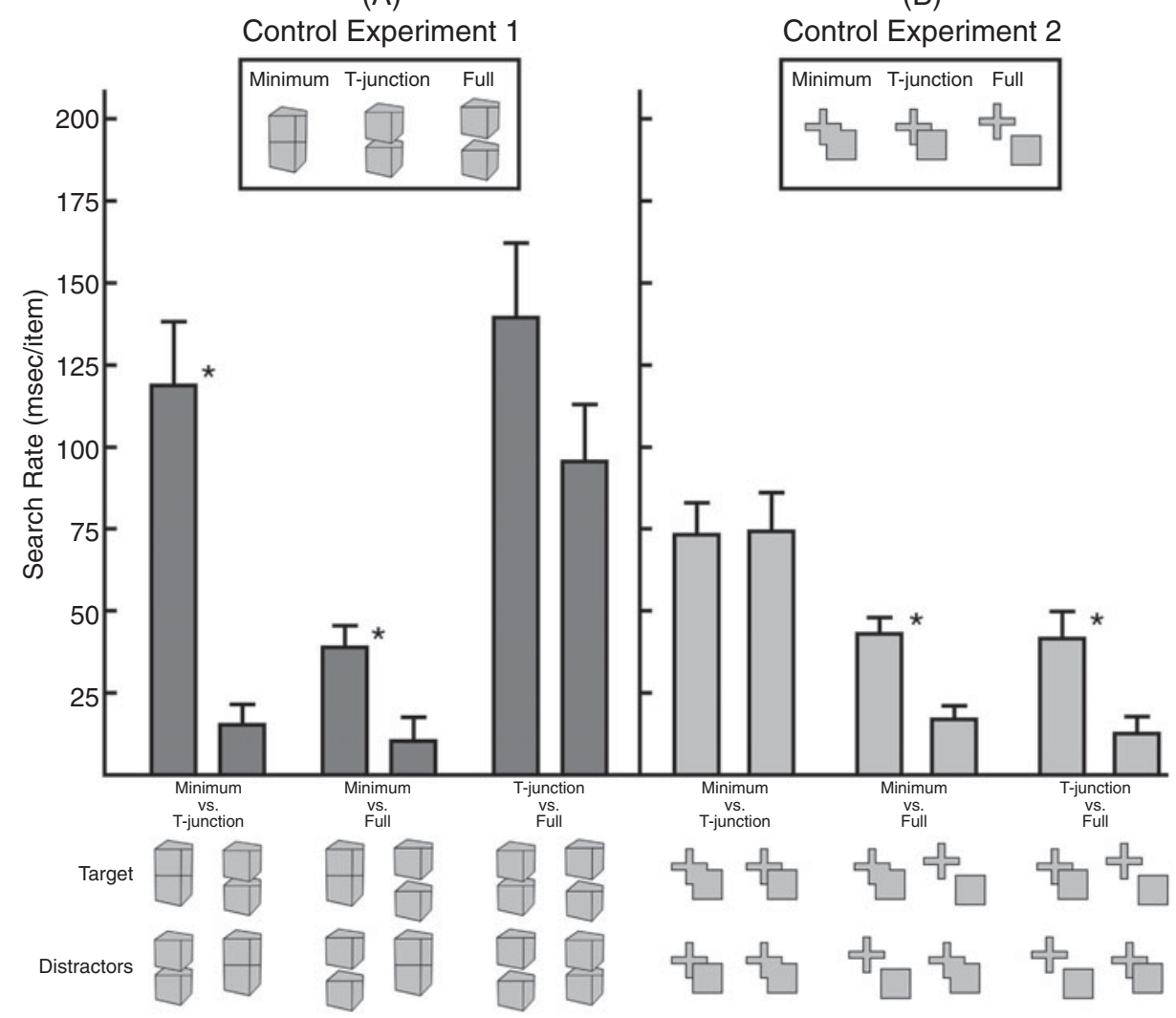

Figure 6. Bar graphs of the mean search rates (in milliseconds/item) as a function of targetdistractor combination for the two control experiments. (A) Control Experiment 1. The twodimensional configurations in the main experiment were used, with the exception of the configurations showing intermediate contour segmentation. For these configurations, the L-junctions that were present at the locations of the concavities in the outer contour have been replaced by $T$ junctions. (B) Control Experiment 2. The configuration showing T-junctions is interpreted as a cross juxtaposed to a square. That is, in this case, T-junctions do not lead to an occlusion interpretation. Error bars represent one standard error of the mean. An asterisk placed between a pair of bars indicates a significant difference (i.e., a search asymmetry) between the corresponding targetdistractor combinations.

ure $6 \mathrm{~B}$, the configurations showing T-junctions favored a mosaic interpretation (in which a cross is juxtaposed to a square) over an occlusion interpretation. That is, global figural aspects, such as symmetry, trigger the mosaic interpretation. An advantage of global aspects over local aspects has been shown in various studies on occlusion (e.g., de Wit \& van Lier, 2002; van Lier, van der Helm, \& Leeuwenberg, 1994). By comparing these configurations with the configurations showing minimum and full contour segmentation, segmentation of configurations based on local T-junctions can be further examined.

In this second control experiment, the same task was administered $(N=12)$, and the same data analysis was performed. No search asymmetry was found between configurations showing minimum contour segmentation and those showing T-junctions $(F<1$; Figure 6B, two bars on the left). Search asymmetries were found between configurations showing minimum and those showing full contour segmentation $[F(1,11)=6.65, p<.05$;
Figure 6B, middle two bars], and between configurations showing T-junctions and those showing full contour segmentation $[F(1,11)=6.26, p<.05$; Figure $6 \mathrm{~B}$, two bars on the right]. This indicates that the presence of T-junctions in the target does not necessarily lead to faster identification, as compared with the opposite situation. Thus, T-junctions are not sufficient for relatively fast segmentation of object configurations. The results of both control experiments can be explained by the notion of OB connectedness (Koning \& van Lier, 2003, 2004). That is, an interpretation of the distractors as OB-disconnected elements (i.e., the configurations showing T-junctions in the first control experiment and the configurations showing full contour segmentation in both control experiments) decreases search performance, as compared with the opposite situation.

In conclusion, in three experiments, it was found that interpretations guide segmentation processes. Segmentation based on outer contours and T-junctions does fa- 
cilitate target identification in a visual search display. However, when 3-D information about objects is available in a visual search display, segmentation strongly depends on perceived OB connectedness, more than on IB connectedness. Whereas segmentation processes are often considered to precede object interpretations, the present results indicate that object interpretations guide segmentation processes as well.

\section{REFERENCES}

Biederman, I. (1987). Recognition-by-components: A theory of human image understanding. Psychological Review, 94, 115-147.

Brown, J. M., Weisstein, N., \& May, J. G. (1992). Visual search for simple volumetric shapes. Perception \& Psychophysics, 51, 40-48.

DE Wit, T. C. J., \& VAN LiER, R. J. (2002). Global visual completion of quasi-regular shapes. Perception, 31, 969-984.

Duncan, J., \& Humphreys, G. W. (1989). Visual search and stimulus similarity. Psychological Review, 96, 433-458.

EnNs, J. T., \& Rensink, R. A. (1990). Sensitivity to three-dimensional orientation in visual search. Psychological Science, 1, 323-326.

Hoffman, D. D., \& Richards, W. A. (1984). Parts of recognition. Cognition, 18, 65-96.

Hoffman, D. D., \& Singh, M. (1997). Salience of visual parts. Cognition, 63, 29-78.

Hulleman, J., te Winkel, W., \& Boselie, F. (2000). Concavities as basic features in visual search: Evidence from search asymmetries. Perception \& Psychophysics, 62, 162-174.

Koning, A., \& VAN LIER, R. (2003). Object-based connectedness facilitates matching. Perception \& Psychophysics, 65, 1094-1102.

Koning, A., \& VAN LiER, R. (2004). Mental rotation depends on the number of objects rather than on the number of image fragments. Acta Psychologica, 117, 65-78.

Kunar, M. A., Humphreys, G. W., Smith, K. J., \& Hulleman, J.
(2003). What is "marked" in visual marking? Evidence for effects of configuration in preview search. Perception \& Psychophysics, 65, 982-996.

LORCH, R. F., JR., \& Myers, J. L. (1990). Regression analyses of repeated measures data in cognitive research. Journal of Experimental Psychology: Learning, Memory, \& Cognition, 16, 149-157.

Palmer, S. E. (1999). Vision science. Cambridge, MA: MIT Press.

Rauschenberger, R., Peterson, M. A., Mosca, F., \& Bruno, N. (2004). Amodal completion in visual search: Preemption or context effects? Psychological Science, 15, 351-355.

RENSINK, R. A., \& EnNs, J. T. (1998). Early completion of occluded objects. Vision Research, 38, 2489-2505.

SAIKI, J., \& Hummel, J. E. (1998). Connectedness and the integration of parts with relations in shape perception. Journal of Experimental Psychology: Human Perception \& Performance, 24, 227-251.

Siddiei, K., Tresness, K. J., \& Kimia, B. B. (1996). Parts of visual form: Psychophysical aspects. Perception, 25, 399-424.

Singh, M., Seyranian, G. D., \& Hoffman, D. D. (1999). Parsing silhouettes: The short-cut rule. Perception \& Psychophysics, 61, 636-660.

Treisman, A., \& Gelade, G. (1980). A feature-integration theory of attention. Cognitive Psychology, 12, 97-136.

van Lier, R., VAN Der Helm, P., \& Leeuwenberg, E. (1994). Integrating global and local aspects of visual occlusion. Perception, 23, 883-903.

VAN LIER, R., \& WAGEMANS, J. (1998). Effects of physical connectivity on the representational unity of multi-part configurations. Cognition, 69, B1-B9.

Vecera, S. P., Behrmann, M., \& FilapeK, J. C. (2001). Attending to the parts of a single object: Part-based selection limitations. Perception \& Psychophysics, 63, 308-321.

Xu, Y., \& Singh, M. (2002). Early computation of part structure: Evidence from visual search. Perception \& Psychophysics, 64, 10391054 .

(Manuscript received October 30, 2003; revision accepted for publication October 28, 2004.) 